\title{
Enrichment of open reading frames presented on bacteriophage M13 using Hyperphage
}

\author{
Michael Hust ${ }^{1}$, Maren Meysing1, Thomas Schirrmann1, Martin Selke², Jochen Meens², \\ Gerald-F. Gerlach², and Stefan Dübel ${ }^{1}$
}

BioTechniques 41:335-342 (September 2006)

doi 10.2144/000112225

\begin{abstract}
The enrichment of open reading frames (ORFs) from large gene libraries and the presentation of the corresponding polypeptides on filamentous phage M13 (phage display) is frequently used to identify binding partners of unknown ORFs. In particular, phage display is a valuable tool for the identification of pathogen-related antigens and a first step for the development of new diagnostics and therapeutics. Here, we introduce a significant improvement of phage-based ORF enrichment by using Hyperphage, a helperphage with a truncated gIII. The methods allow both the enrichment of ORFs from cDNA libraries and the display of the corresponding polypeptides on phage, thus combining ORF enrichment with a screening for binding in one step without any further subcloning steps. We demonstrated the benefits of the method by isolating the sequences encoding two predicted immunogenic epitopes of the outer membrane protein D encoding gene (ompD) of Salmonella typhimurium. Here, we showed that when using a mixture of three constructs with only one containing an ORF, solely this correct construct could be reisolated in phage particles. Further, both epitopes were detected by enzyme-linked immunosorbent assay (ELISA), demonstrating correct translation of fusion proteins. Furthermore, the enrichment system was evaluated by the enrichment of ORFs from total cDNA of lymphocytes. Here, we could show that $60 \%$ of the phage contained ORFs, which is an increase of an order of magnitude compared with conventional phage expression system. Together, these data show that the Hyperphage-based enrichment system significantly improves the enrichment of ORFs and directly allows the display of the corresponding polypeptide on bacteriophage M13.
\end{abstract}

\section{INTRODUCTION}

Phage display was initially described by G.P. Smith (1) as a powerful tool for the presentation of recombinant polypeptides on filamentous phage M13 and the selection of the encoding sequences from expression libraries. The genotype and phenotype of polypeptides are linked by fusing the corresponding gene fragments to the phage gene gIII encoding the minor coat protein III (pIII) of the filamentous bacteriophage M13. At first, the gene fragments were directly inserted into the phage genome fused to the wild-type gIII gene. Today, most successful phage display systems uncouple polypeptide expression from phage propagation by providing the genes encoding the polypeptide::pIII fusion proteins on a separate plasmid (phagemid), containing a phage morphogenetic signal for packaging into the assembled phage particles (2). A large variety of phagemids has been constructed for M13 phage display (for an overview of phagemids used for the display of antibody fragments see Reference 3$)$. The in vitro procedure for isolating polypeptides or peptides by their binding activity was called panning (4).

The display and selection of antibody fragments currently is the major application of phage display technology. However, another important field is the presentation of polypeptides encoded by cDNA or genomic DNA. This approach was used for the identification of allergens (5), identification of binding epitopes of antibodies (6), identification of ligands in protein-ligand interactions (7), or identification of tumor polypeptides eliciting immune responses (8) among others. For an overview of phage displayed polypeptides derived from
cDNA, see Reference 9. Phage displaybased methods can also be used in highthroughput setup for parallel selection of binders $(10,11)$.

A disadvantage of current approaches to phage-based polypeptide expression results from the necessity of the in-frame insertion between signal sequence and the gIII of the phage to obtain expression. As a consequence, only $6.25 \%$ - the rate of nondirectional cloning - of the cloned DNA fragments represent true open reading frames (ORFs). Furthermore, stop codons in the gene fragments can abrogate the translation of the gene::gIII fusion required for the enrichment. Due to the selection pressure, phage display vectors without inserted fragments or with defective inserts are more efficiently propagated than vectors containing a substantial ORF insert. This effect further hampers the selection of binders by increasing the number of junk clones in a

1Technische Universität Braunschweig, Braunschweig and 2Stiftung Tierärztliche Hochschule Hannover, Hannover, Germany 


\section{Research Reports}

selection to $90 \%-99 \%$. Consequently, it would be helpful to enrich for ORFs. Therefore, the gene fragments were cloned as fusions with the ampicillin resistance $\beta$-lactamase gene to promote the enrichment of gene fragments that are in-frame with the selection marker (12). However, a combination of ORF enrichment based on the ampicillin resistance gene with presentation of the corresponding polypeptide on phage requires the removal of the $\beta$-lactamase gene fragment after antibiotic enrichment. This removal was achieved either by subcloning of the ORF fragment (13) or by constructing a $\beta$ lactamase gene fragment flanked by loxP sites that could then be removed in vivo by Cre recombinase (14). Both systems still require subcloning of the ORF-enriched libraries before further functional selection, thus leading to a loss of complexity.

Here, we present a novel method for the enrichment of ORFs combined with the presentation of the corresponding polypeptides on M13 phage. The helperphage Hyperphage (15) has a truncated gIII on the phage genome, so that the pIII fusion protein encoded on the phagemid is the only source of intact pIII, which is an essential coat protein for the assembly of infective phage. The resulting phage contain only phagemid encoded polypeptide:: pIII fusion, thereby providing oligovalent display. In contrast, when using M13K07 for packaging, two sources of pIII are available, and only $1 \%-10 \%$ of the phage display a polypeptide (15). In the Hyperphage-based enrichment system, the DNA fragments are cloned upstream of gIII and packaged with Hyperphage. Only if the DNA fragment is cloned in-frame with the pelB leader sequence and gIII, functional polypeptide::pIII fusion protein can be produced and consequently functional phage can be assembled. Further, the encoded polypeptides are displayed on the phage particles without any further cloning step, avoiding loss of library diversity.

We provide the proof of principle for this method by showing the enrichment of the correct frame and the expression of two antigenic epitopes from the Salmonella enterica subspecies enterica serovar typhimurium (Salmonella typhimurium) ompD gene and by expression of ORFs from lymphocytederived cDNA.

\section{MATERIALS AND METHODS}

\section{Construction of pHORF2 and Cloning of the ompD Gene Fragments ant11 and ext 3}

Standard cloning procedures were performed according to Reference 16. The $\mathrm{pHORF} 2$ vector was derived from the phagemid vector pHAL1 (17). Here, the Fab expression cloning cassette between NheI and NotI was replaced with a cassette containing an additional NotI site for the cloning of cDNA fragments. Three ochre stop codons in different reading frames were inserted between the NotI sites to prevent expression of functional pIII in religations. The sequence encoding the strep tag II (18) between NotI and BamH1 was replaced by a sequence encoding a $\mathrm{His}_{6}$ tag. Putative antigenic regions and surface-exposed loops were predicted using the HUSAR software package program, antigenic (19) (genius.dkfzheidelberg.de) and the HMM-B2TMR program (gpcr.biocomp.unibo.it) (20), respectively. The $o m p D$ gene fragments, antigenic11 (antl1) and external3 (ext3), were amplified from plasmid pSOM500, an expression vector based on pGEX-5X-3 (Amersham Bioscience, Darmstadt, Germany) containing the mature part of the OmpD protein (GenBank ${ }^{\circledR}$ accession no. NC003197, position 1655236-1656261), fused to gluthathion-S-transferase (Selke, unpublished data) and cloned into the NotI site of pHORF2. Transformations of XL1Blue $\mathrm{MRF}^{\prime}$ (Stratagene, Amsterdam, The Netherlands) with these constructs were done by electroporation according to the manufacturer's instructions. All affected regions of the constructs were comfirmed by DNA sequencing using an ABI PRISM ${ }^{\circledR} 310$ sequencer (Applied Biosystems, Foster City, CA, USA).

\section{Production of Polypeptide Phage}

Fifty milliliters $2 \times$ tryptone yeast extract (TY) medium (16) containing $100 \mu \mathrm{g} / \mathrm{mL}$ ampicillin and 100 $\mathrm{mM}$ glucose were inoculated with an overnight culture of pHORF2 constructs bearing bacteria to an absorbance at $600 \mathrm{~nm}\left(\mathrm{~A}_{600}\right)$ of 0.025 . The bacteria were grown to an $\mathrm{A}_{600}$ of $0.4-0.5$ at $37^{\circ} \mathrm{C}$ and $250 \mathrm{rpm}$. Two milliliters Escherichia coli Top 10F (Invitrogen, Karlsruhe, Germany) (approximately $1 \times 10^{9}$ bacteria) were infected with $2 \times 10^{10}$ helperphage M13K07 (21) or Hyperphage (15), incubated at $37^{\circ} \mathrm{C}$ for $30 \mathrm{~min}$ without shaking, followed by $30 \mathrm{~min}$ at 250 $\mathrm{rpm}$. The infected cells were harvested by centrifugation for $10 \mathrm{~min}$ at $3220 \times$ $g$. The pellet was resuspended in 30 $\mathrm{mL} 2 \times$ TY containing $100 \mu \mathrm{g} / \mathrm{mL}$ ampicillin and $50 \mu \mathrm{g} / \mathrm{mL}$ kanamycin. The phage were produced at $30^{\circ} \mathrm{C}$ and $250 \mathrm{rpm}$ for $16 \mathrm{~h}$. Cells were pelleted by centrifugation for $20 \mathrm{~min}$ at $3220 \times$ $g$. The phage in the supernatant were precipitated with $1 / 5$ volume of $20 \%$ (w/v) polyethylene glycol (PEG)/2.5 M $\mathrm{NaCl}$ solution for $1 \mathrm{~h}$ on ice with gentle shaking and pelleted by centrifugation for $1 \mathrm{~h}$ at $3220 \times g$ at $4^{\circ} \mathrm{C}$. The precipitated phage were resuspended in 300 $\mu \mathrm{L}$ phosphate-buffered saline (PBS) (16), and cell debris was pelleted by additional centrifugation for $5 \mathrm{~min}$ at $15,400 \times g$ at $20^{\circ} \mathrm{C}$. The supernatant containing the polypeptide phage were stored at $4^{\circ} \mathrm{C}$. Phage titration (colonyforming units; cfu) was done according to Reference 22 with one modification; namely the infected bacteria were pipeted directly onto LB agar plates, omitting the nitrocellulose sheets.

\section{Sodium Dodecyl Sulfate Polyacrylamide Gel Electrophoresis and Immunoblot}

Phage proteins were separated by reducing sodium dodecyl sulfate polyacrylamide gel electrophoresis (SDS-PAGE) and blotted onto a polyvinylidene fluoride (PVDF) membrane. The membrane was blocked with 5\% (w/v) skimmed milk powder (Roth, Karlsruhe, Germany) in PBST (PBS plus $0.1 \%$, v/v, Tween ${ }^{\circledR 20}$ ) for $1 \mathrm{~h}$ at room temperature. The minor coat protein $\mathrm{pIII}$ was detected with mouse monoclonal antibody (MAb) antipIII (Mobitec, Göttingen, Germany) (1:2000) for $1.5 \mathrm{~h}$ at room temperature, followed by washing twice with PBST. Goat anti-mouse (Fc-specific; Sigma, 
Taufkirchen, Germany) (1:2000) conjugated with alkaline phosphatase (AP) was used for detection and visualized by nitroblue tetrazolium/5-bromo-4chloro-3-indolyl-phosphate (NBT/ BCIP).

\section{Enzyme-Linked Immunosorbent Assay}

Microtiter plates (Costar, Cambridge, MA, USA) were coated with dilutions of OmpD polypeptide displaying phage in $100 \mu \mathrm{L}$ PBS/well overnight at $4^{\circ} \mathrm{C}$. Coated wells were washed three times with PBS and blocked with $2 \%(\mathrm{w} / \mathrm{v})$ skim milk powder in PBST (MPBST) for $1.5 \mathrm{~h}$ at room temperature, followed by washing three times with PBS. The hyperimmune serum, 93B4, was derived from pigs immunized with the live vaccine Salmoporc ${ }^{\circledR}$ (Impfstoffwerk Dessau-Thornau, Berlin, Germany), and Derby was a pool of convalescent sera from pigs infected with $S$. enterica subspecies enterica serotype Derby (kindly provided by Josef Kamphues, Stiftung Tierärztliche Hochschule Hannover, Germany). The rabbit serum raised against $\mathrm{OmpD}$ was produced by SeqLab GmbH (Göttingen, Germany). All sera were diluted 1:2000 in 100 $\mu \mathrm{L}$ MPBST and incubated for $1.5 \mathrm{~h}$, followed by washing five times with PBST. Bound serum antibodies were detected with goat anti-pig sera conjugated with horseradish peroxidase (HRP; 1:10000) or goat anti-rabbit sera conjugated with HRP $(1: 10000)$, respectively. The immunostain was visualized with trimethyl benzidine (TMB) substrate (Bio-Rad Laboratories, München, Germany). The staining reaction was stopped by adding $100 \mu \mathrm{L} 1 \mathrm{~N}$ sulfuric acid. The absorbances at 450 and $620 \mathrm{~nm}$ were recorded using a Sunrise ${ }^{\mathrm{TM}}$ microtiter plate reader (Tecan, Crailsheim, Germany). The absorbance of scattered light at $620 \mathrm{~nm}$ was subtracted from the absorbance at $450 \mathrm{~nm}$.

\section{Cloning of cDNA Fragments into pHORF2}

Lymphocytes were isolated from blood samples of 44 donors with LymphoPrep $^{\mathrm{TM}}$ (Progen, Heidelberg,
Germany) according to the manufacturer's instructions. The total RNA was isolated using TRIzoL ${ }^{\circledR}$ (Invitrogen) according to the manufacturer's instructions. Total RNA from human lymphocytes was reverse transcribed to cDNA using SuperScript ${ }^{\mathrm{TM}}$ II reverse transcriptase (Invitrogen) using random hexameric oligonucleotide primers according to the manufacturer's instructions. To the total reaction volume of the cDNA synthesis $(20 \mu \mathrm{L}), 40 \mathrm{U} E$. coli DNA polymerase I, $1 \mathrm{U}$ RNase $\mathrm{H}, 5 \mathrm{U}$ E. coli ligase, $0.2 \mathrm{mM}$ dNTPs each, $1 \times E$. coli ligase buffer, and distilled water were added to a final volume of $75 \mu \mathrm{L}$ (all enzymes are from New England Biolabs, Frankfurt, Germany). The reaction was incubated at $16^{\circ} \mathrm{C}$ for $3 \mathrm{~h}$ and afterwards purified with the GFX ${ }^{\mathrm{TM}}$ DNA and Gel Band Purification kit (Amersham Bioscience) according to the manufacturer's instructions. One microgram double-stranded DNA fragments was polished using $0.1 \mathrm{mM}$ dNTPs each, $2.5 \mathrm{U}$ T4-DNA polymerase, $1 \times$ T4-DNA polymerase buffer, and distilled water, added to a final volume of $20 \mu \mathrm{L}$ (all enzymes are from MBI Fermentas, St. Leon-Rot, Germany). The reaction was incubated for $20 \mathrm{~min}$ at $11^{\circ} \mathrm{C}$, inactivated at $70^{\circ} \mathrm{C}$ for $10 \mathrm{~min}$, and purified with the GFX DNA and Gel Band Purification kit. One microgram vector $\mathrm{pHORF} 2$ was digested with $0.5 \mathrm{U}$ NotI (MBI Fermentas) in a $20-\mu \mathrm{L}$ reaction volume for $3 \mathrm{~h}$ at $37^{\circ} \mathrm{C}$. Afterwards, $0.5 \mathrm{U}$ calf intestinal phosphatase (MBI Fermentas) was added and incubated for an additional $30 \mathrm{~min}$. This step was repeated once. The vector was purified using the GFX DNA and Gel Band Purification kit. Polished cDNA fragments were cloned into $100 \mathrm{ng}$ of the polished and dephosporylated pHORF2 in a molar ratio of 3:1 using $1 \mathrm{U}$ T4 ligase (Promega, Mannheim, Germany). The ligation reaction was precipitated with ethanol and sodium acetate, and the pellet was washed two times with $70 \%$ ethanol. Transformations of Top $10 \mathrm{~F}^{\prime}$ with the constructs were done by electroporation according to the manufacturer's instructions and plated onto $2 \times \mathrm{TY}$ agar plates supplemented with $100 \mu \mathrm{g} / \mathrm{mL}$ ampicillin and $100 \mathrm{mM}$ glucose $(2 \times \mathrm{TY}-$ GA; 15-cm Petri dish). Colonies were harvested by suspending in $2.5 \mathrm{~mL} 2 \times$
TY-GA with a Drigalsky spatula. One hundred microliters of the harvested bacteria were used for phage packaging using Hyperphage (see above).

\section{RESULTS}

\section{Cloning of ompD Gene Fragments into pHORF2}

The vector $\mathrm{pHORF} 2$ was constructed in order to clone DNA fragments into its NotI sites for the enrichment of ORFs consisting of the pelB leader, the cloned DNA fragments, and the gIII gene and to display the corresponding peptides as gIII fusions (Figure 1A).

For the evaluation of the enrichment system using Hyperphage, two fragments of the S. typhimurium ompD gene were cloned into pHORF2. In silico analysis revealed the presence of 14 putative immunogenic regions (Ant1-Ant14) and 8 surface exposed external loops (Ext1-Ext8) within the OmpD protein. The largest polypeptides, Ext3 ( $\left.\mathrm{Y}^{121}-\mathrm{R}^{148}\right)$ and Ant11 $\left(\mathrm{F}^{276}-\mathrm{S}^{297}\right)$, were selected for further characterization. The corresponding gene fragments-with minor discrepancies to the predicted sequence due to cloning reasons-ext3 (coding region $\mathrm{D}^{107}-\mathrm{D}^{143}$ ) and ant11 (coding region E $^{261}-S^{297}$ ) were cloned into pHORF2 in three different reading frames. In frame 3 phagemids, the gene fragments are in-frame with the gIII, resulting in the expression of a functional Ext3/ Ant11::pIII fusion protein (Figure 1B). Frame1 and frame2, phagemids have -1 or +1 frameshifts, respectively, in the $5^{\prime}$ end of the gene fragment and contain one or more ochre (TAA) or opal (TGA) stop codons in the subsequent sequence, which should abolish the production of functional pIII.

\section{Enrichment of In-Frame ompD Gene Fragments}

Each of the six phagemids was packaged with M13K07 or with Hyperphage. Furthermore, an equal mixture of all three ext 3 or ant11 phagemid-bearing bacteria were packed with both helperphage types. As expected, when packaging with M13 K07, all titers-about $2 \times 10^{13} \mathrm{cfu} /$ 


\section{Research Reports}

$\mathrm{mL}$-were similiar. In contrast, when using Hyperphage, the titers of out-offrame phagemids were significantly lower with a titer of about $3 \times 10^{7} \mathrm{cfu} /$ $\mathrm{mL}$ for frame 1 and $5 \times 10^{6} \mathrm{cfu} / \mathrm{mL}$ for frame 2 in comparison to the in-frame phagemid frame 3 with a titer of $2.7 \times$ $10^{12} \mathrm{cfu} / \mathrm{mL}$ (Figure 2). The phagemid mixture was packaged as well as the frame 3 phagemid. The results for the gene fragment ant11 were similiar. Randomly selected clones from the titer plates of the six different phagemids packed with Hyperphage were sequenced. All clones derived from the phage containing frame1, frame2, and frame 3 phagemids contained the original phagemid. Clones derived from the mixture experiment were sequenced $(n=25)$, showing that all phage were bearing a frame 3 plasmid, showing an efficient enrichment of the correct ORFs by the system.

\section{Display of OmpD Polypeptides on Phage}

We further analyzed whether the OmpD polypeptides were presented on the phage when Hyperphage or M13K07 was used for packaging. The proteins of the phage particles produced were separated by SDS-PAGE, Western blotted, and the pIII was immunostained (Figure 3). When using Hyperphage or packaging, both oligopeptides were well presented on the phage surface. In contrast to Hyperphage when using M13K07, no presentation was detectable in immunoblot. Thus, the display of the polypeptide on phage using pHORF2 and Hyperphage for packaging is possible.

\section{Analysis of the Antigenic Character of the OmpD Polypeptides Using ELISA}

The OmpD polypeptides Ext3 and Ant11 were predicted to be immunogenic $S$. typhimurium antigens. An enzyme-linked immunosorbent assay (ELISA) using the phage as antigen and immune sera for detection was used to check both the prediction algorithm and the correct surface presentation of the antigenic polypeptide on phage. Ext 3 or Ant 11 polypeptide presenting

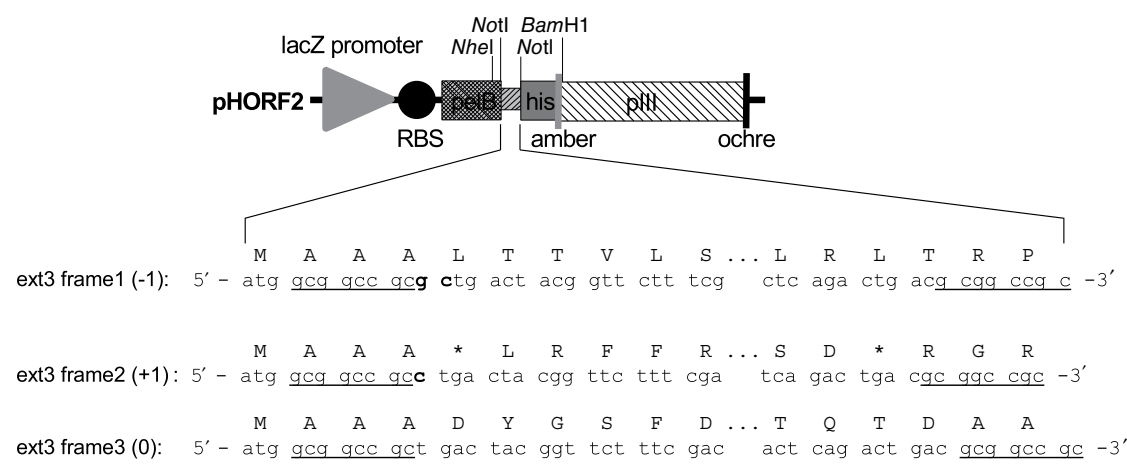

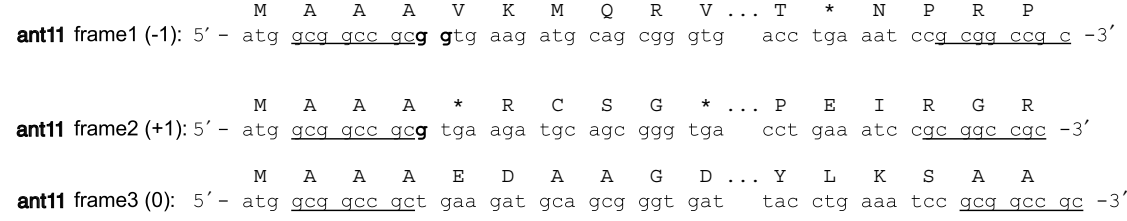

Figure 1. The phage vector construct pHORF2. (A) View of the expression cassette. (B) pHORF2 variants used in this study. The NotI sites are underlined. The additional base pairs in the frame1 and frame 2 constructs are marked in bold. The asterisk * indicates a stop codon. Abbreviations: lacZ promoter, promoter of the bacterial lac operon; RBS, ribosome binding site; pelB, signal peptide sequence of bacterial pectate lyase Erwinia caratovora, mediating secretion into the periplasmic space; ochre, ochre stop codon; pIII, gene coding for the phage protein III; amber, amber stop codon; his, 6 histidin residues; terminator, sequence terminating transcription. The elements of the inserts are not drawn to scale.

phage were coated to microtiter wells and detected with porcine sera 93B4 (hyperimmune serum), Derby (convalescent serum), and a rabbit anti-OmpD serum, respectively (Figure 4). The serum 93B4 and the rabbit serum bound both antigens, whereas the Derby serum showed no significant binding to either of the two antigens. Thus, polypeptides presented on phage surface using Hyperphage and pHORF2 can be detected by the corresponding sera, demonstrating correct phage display.

\section{Cloning and Enrichment of cDNA Fragments}

To investigate whether packaging using Hyperphage enhances the yield of selected cDNA fragments in-frame with the leader and gIII, we cloned cDNA derived from total RNA of human lymphocytes into pHORF2. The resulting phagemids were packaged with Hyperphage. Randomly picked clones $(n=47)$ from the titer plates were sequenced to analyze the inserts. The selected inserts had a length of approximately 50-230 bp. As expected, due to the use of total RNA, the selected ORFs includes a high proportion of inserts derived from ribosomal RNA. Sixty percent of the inserts were inframe with the pelB leader and the gIII, and $4 \%$ were in-frame but contained a stop codon in the sequence of the cloned insert. Thirty percent of the cloned inserts were not in-frame with the pelB leader and the gIII, and $6 \%$ were out-offrame and contained an additional stop codon. Taken together, the phagemid packaging using Hyperphage leads to 10-fold enrichment of ORFs from theoretically $6 \%$ to $60 \%$ of all cloned inserts.

\section{DISCUSSION}

The enrichment of cDNAs and the presentation of the corresponding polypeptides on bacteriophage M13 are essential tools to identify binding partners in biological systems (for a review see Reference 9). After cloning cDNA or genomic DNA into phage display vectors, only about $6 \%$ of the obtained phagemids contain a complete ORF consisting of the pelB leader, the cloned DNA insert, and the gIII. As a result, subsequent panning has to be done with a phage library 
A

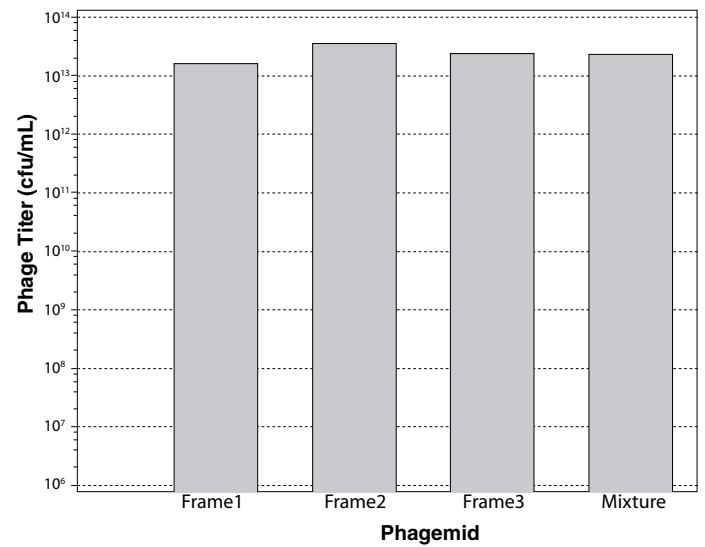

B

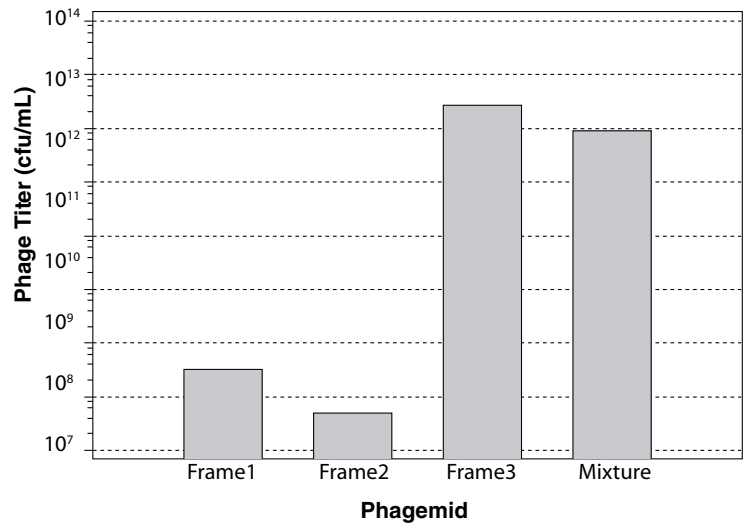

Figure 2. Phage titer obtained from a 30-mL culture of TOP $10 F^{\prime}$ transformed with each pHORF2 ext3 phagemid construct or of the bacteria mixture containing all three different phagemids. Phage packaged with (A) M13K07 and (B) with Hyperphage. cfu, colony-forming units.

containing more than $90 \%$ of junk clones. Every phage display selection is a single molecular binding event in a background of $10^{8}-10^{11}$ nonspecific molecules. Consequently, there is tremendous sensitivity of phage display to low signal-to-noise ratio. As a consequence, a contamination of the panned library with $>90 \%$ junk sequences dramatically hampers the panning success. Thus, a phage display system is desirable that combines the enrichment of correct ORFs with the display of the corresponding polypeptide on phage without additional efforts like subcloning of the selected ORF.

In this study, we developed a system for the enrichment of ORFs from phagemid-cloned cDNA fragments and the display of the corresponding polypeptides on bacteriophage M13 using the helperphage Hyperphage (15). In contrast to the helperphage M13K07 (21), in which only 1\%-10\% of the phage particles display the phagemid-encoded polypeptide, the use of Hyperphage results in polyvalent display on almost every single phage particle. The Hyperphage genome contains a truncated gIII. Therefore, only one source of pIII-derived from the polypeptide::pIII fusion on the phagemid-is available, in contrast to M13K07, in which pIII from two different sources compete for the phage assembly (Reference 15 and unpublished data). The idea for the enrichment of ORFs using Hyperphage is based upon the hypothesis that bacteria-bearing phagemids without a consecutive ORF consisting of the
pelB leader sequence, the cloned insert, and gIII-encoding the corresponding polypeptide::pIII fusion protein-will not produce intact phage particles. This was successfully demonstrated for two polypeptide fragments from antigenic regions of OmpD of S. typhimurium. As expected, the correct frame phagemids were packaged about $10^{5}$ times more efficiently than the incorrect frame phagemids when using Hyperphage, whereas with M13K07, the titers for all frames were similar. The titers from the packaged phagemids of the frame1, frame 2 and frame 3 bacteria mixture are analogous to the frame 3 phagemid titers. All sequenced clones from the mixture had a correct frame insert. Thus, the enrichment system for ORFs using Hyperphage works.

The polypeptides Ext3 and Ant11 encoded by the pHORF2 frame 3 constructs were analyzed by immunoblot to evaluate their display on phage. Here, both polypeptides were presented efficiently when using Hyperphage for packaging, whereas when using M13K07 for packaging, no fusion::pIII band was detectable. This is in concordance with former packaging with Hyperphage. The quantity of the presentation of the fusion proteins depend on the fusion protein and the individual packaging. Often when using Hyperphage, a wild-type pIII band is detectable due to residual proteolytic degradation $(15,17)$. The antigenic character of both OmpD polypeptides was verified by ELISA with serum from infected or immunized animals. The antigen Ext 3 was also detected better than Ant11, presumbly due to the better display of Ext3. Thus, the displayed antigens can be detected by the appropriate sera.

The pHORF2 phage vector has an amber stop codon between the His tag coding region and the gIII. $E$. coli supE44 strains, like XL1-Blue $\mathrm{MRF}^{\prime}$, which express a suppressor transfer RNA (tRNA) binding to TAG codons and incorporating glutamine during translation, are able to produce the polypeptide::pIII fusion protein coded by the pHORF2 phagemid. In contrast to XL1-Blue $\mathrm{MRF}^{\prime}$, the TOP $10 \mathrm{~F}^{\prime}$ strain, containing no suppressor tRNA mutation, should not produce any polypeptide::pIII fusion protein. Accordingly, no infectious phage particle should be produced by the TOP $10 \mathrm{~F}^{\prime} E$. coli bacteria if Hyperphage is used for the packaging of pHORF2 phagemids. However, in practice, phage titers were only about 10 -fold lower. We first investigated the enrichment system using Hyperphage with XL1-Blue MRF' (data not shown). The differences of phage titers for the different readings frames of ant 11 and ext 3 in pHORF 2 separately packaged with Hyperphage and M13K07 for each frame are similar in XL1-Blue MRF' and TOP $10 \mathrm{~F}^{\prime}$, despite the fact that phage titers obtained with the TOP $10 \mathrm{~F}^{\prime}$ bacteria were generally about 10 times lower compared with XL1-Blue MRF'. XL1-Blue MRF' clones containing phagemids of all three reading frames of the respective ompD gene fragment were combined to a mixture. After packaging this 


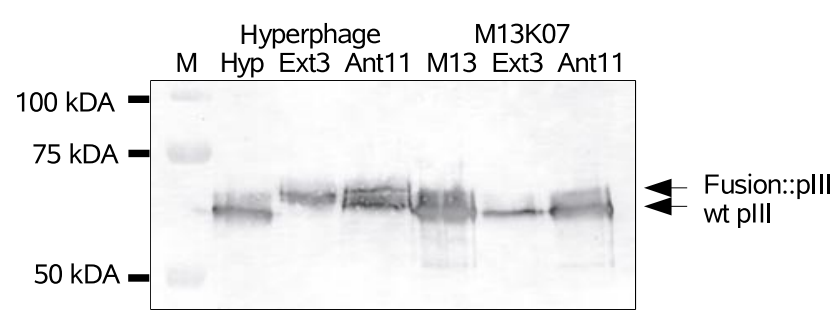

Figure 3. Biochemical analysis of the presentation of the Ext3 and Ant11 polypeptide on phage produced with Hyperphage or M13K07. $10^{10}$ phage/lane were separated on a reducing $10 \%$ sodium dodecyl sulfate polyacrylamide gel electrophoresis (SDS-PAGE). pIII was detected using mouse monoclonal antibody (MAb) anti-pIII. Abbreviations: Hyp, the helperphage Hyperphage (wild-type pIII control); M13, M13K07 (wildtype pIII control); Ext3, phage displaying the Ext3 polypeptide; Ant11, phage presenting the Ant11 polypeptide; wt, wild-type.

mixture with Hyperphage, produced phage clones were analyzed by DNA sequencing. Unexpectedly, we found all three reading frames. To investigate this effect, we performed additional studies. We found that the occurrence of the out-of-frame phagemids frame 1 and frame 2 was time-dependent. One hour after starting the phage production, only frame 3 phagemid-bearing phage were produced. The amount of frame 1 and frame 2 phagemids containing phage particles increased step-by-step within the next hours. We suspected a horizontal gene transfer from frame 3 phagemid-bearing bacteria to frame 1 and frame 2 phagemid-bearing bacteria by either re-infection or conjugation. In further experiments, we were able to exclude re-infection as well as conjugation being responsible for this phenomenon (data not shown). We also used another E. coli strain with a supE44 genotype, TG1, and another nonsuppressor strain HB2151. Here, the results were comparable to XL1Blue MRF'. Only the TOP $10 \mathrm{~F}^{\prime} E$. coli strain showed no horizontal gene transfer and allowed the enrichment of ORFs using Hyperphage.

Faix et al. (13) used the fusion of the cloned cDNA with the $\beta$-lactamase gene for the enrichment of ORFs. However, in this system, it is necessary to subclone the selected inserts into a phage display vector. Zacchi et al. (14) also used a $\beta$-lactamase fusion for the enrichment. In this system, the $\beta$ lactamase is flanked by lox $P$ sites and must be removed by Cre recombinase before allowing the display of the polypeptide encoded by DNA fragment on phage. They tested their system by cloning of the antilysozyme antibody D1.3 encoding gene fragment in-frame or out-of-frame to the $3^{\prime}$ end of the $\beta$-lactamase gene. After removal of the $\beta$-lactamase gene, the majority of phagemid clones displayed antibody fragments that were able to bind their antigen. Without removal of the $\beta$ lactamase gene, however, the D1.3 presenting phage could not bind to lysozyme. The novel Hyperphagebased ORF enrichment described in the study does not require an additional cloning step in contrast to both antibiotic ORF enrichment systems. Due to the fact that each transformation step reduces the diversity of gene libraries, the Hyperphage system will facilitate the cloning of highly diverse cDNA libraries.

The selective enrichment of ORFs using Hyperphage was then tested with a true library of cDNA of human lymphocyte total RNA into pHORF2. As revealed by DNA sequencing, about $60 \%$ of the obtained phagemids had a correct ORF. The other DNA insertion fragments were not in-frame with the gIII or were in-frame and contained a stop codon in the cloned DNA fragment. This is not too surprising, as it already has been shown previously that, after selection by phage display, gene fragments encoding oligopeptides or polypeptides frequently contain frameshifts (23). In spite of that, particularly in the case of +1 frameshifts, these oligopeptides or polypeptides are still displayed on phage particles with the same amino acid sequence like the corresponding constructs without frameshift. The occurrence of RNA secondary structure was suggested to explain this effect. Another explanation could be the selection pressure against oligopeptides or polypeptides, which are toxic for the E. coli host (24).

The antibiotic ORF enrichment described by Faix et al. (13) accomplished an enrichment rate of $97 \%$ ORFs. However, after subcloning in the phage display vector, the percentage of ORFs decreased to $70 \%$. After packaging the phagemids with hyperphage for polyvalent display, the percentage of ORFs increased again to $87 \%$, supporting our data that Hyperphage enhances the enrichment of ORFs. The enrichment of ORFs using the cre/lox $\beta$-lactamase system has not been validated for the cloning of ORFs from total cDNA (14). So, when compared with all existing systems of ORF enrichment, the new method described in this study, the combination of a powerful ORF enrichment with

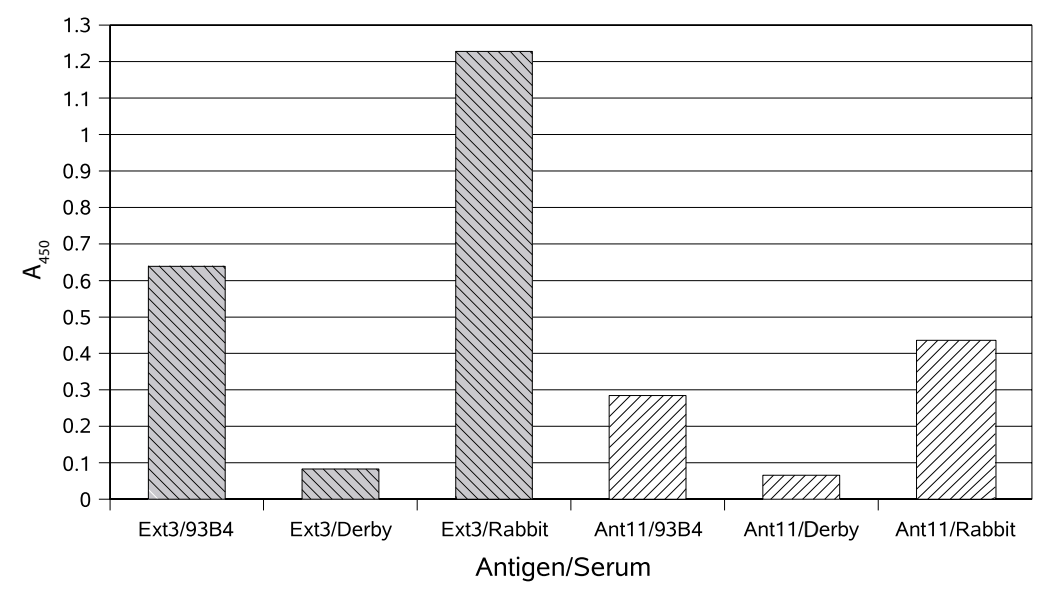

Figure 4. Representative antigen binding enzyme-linked immunosorbent assay (ELISA) with the porcine sera 93B4, Derby, and rabbit anti-OmpD on the polypeptide Ext3 and Ant11 presenting phage. Antigen phage $\left(2 \times 10^{8}\right)$ were coated. The antigenic OmpD fragments were detected using goat anti-pig sera conjugated with horseradish peroxidase (HRP; 1:10000) and goat anti-rabbit sera conjugated with HRP (1:10000), respectively. The background signal of coated Hyperphage incubated with the appropriate serum and detected with the enzyme-conjugated antibodies was subtracted. 
phage display, avoids the adverse effect of library subcloning.

In common phage display systems, the polypeptides are fused to pIII. The polypeptide::pIII fusion protein is transported to the periplasm and folded in the oxidizing milieu of the periplasm. This system is well adapted for antibodies (25). For many proteins, a folding in the reducing milieu of the cytoplasm and the transport of the folded polypeptides via the Tat-pathway are preferable. However, the pIII must be transported unfolded by the Secpathway to the periplasm, where the phage assembly occurs. The twin argine transclocation phage display (TPD) system, basing on the Jun-Fos system, allows the display of polypeptides folded in the bacterial cytoplasm on phage. Here, pIII and polypeptides are fused to cystein-modified forms of helical moieties of Jun and Fos, respectively $(26,27)$. Both fusion proteins are transported separately into the periplasm, where they will be linked via Jun-Fos heterodimerization and disulfide formation $(27,28)$. The combination of our Hyperphage-based ORF enrichment and the TPD system may well enhance the phage display of polypeptides with low probability of correct folding in the periplasm.

Surprising effects like the suspected horizontal gene transfer in our ORF enrichment system with most of the examined E. coli strains and the skipping of frameshifts and stop codons within the gIII fusion gene during phage display shows that bacterial systems are still not fully understood. The mechanisms behind these phenomena must be further investigated to solve problems of the E. coli phage display system, which is an important biotechnological tool for the selection of antibodies and polypeptides.

Taken together, the Hyperphagebased ORF enrichment system allows the selective packaging of gene fragments from cDNA or other DNA sources and the display of the corresponding polypeptide on phage in one step without any further subcloning steps. The presented Hyperphage-based enrichment system will be useful for the selection of antigens from pathogens; selected antigens subsequently could be used for the development of new or improved diagnostic tests and vaccines in human and veterinary medicine. In addition, the method can be expected to greatly improve protein interaction studies of unknown gene pools in functional genomics.

\section{ACKNOWLEDGMENTS}

We gratefully acknowledge the financial support by the German Ministry of Education and Research (BMBF, SMP "Antibody Factory" in the NGFN2 program) and of the German Research Foundation (DFG, SFB 578). M.S. and J.M. were supported by the Bioprofile project PTJ-BIO/0313037. We would thank Josef Kamphues for providing the Derby serum. We would like to thank Saskia Helmsing for technical assistance.

\section{COMPETING INTERESTS STATEMENT}

The authors declare no competing interests.

\section{REFERENCES}

1.Smith, G.P. 1985. Filamentous fusion phage: novel expression vectors that display cloned antigens on the virion surface. Science 228:1315-1317.

2. Breitling, F., S. Dübel, T. Seehaus, I. Kleewinghaus, and M. Little. 1991. A surface expression vector for antibody screening. Gene 104:147-153.

3. Hust, M. and S. Dübel. 2005. Phage display vectors for the in vitro generation of human antibody fragments, p. 7195. In R. Burns (Ed.), Immunochemical Protocols, vol. 295, Methods in Molecular Biology, 3rd ed. Humana, Totowa.

4.Parmley, S.F. and G.P. Smith. 1988. Antibody selectable filamentous fd phage vectors: affinity purification of target genes. Gene 73:305-318.

5. Rhyner, C., M. Weichel, S. Fluckiger, S. Hemmann, T. Kleber-Janke, and R. Crameri. 2004. Cloning allergens via phage display. Methods 32:212-218.

6.Palzkill, T., W. Huang, and G.M. Weinstock. 1998. Mapping protein-ligand interactions using whole genome phage display libraries. Gene 221:79-83.

7. Cochrane, D., C. Webster, G. Masih, and J. McCafferty. 2000. Identification of natural ligands for $\mathrm{SH} 2$ domains from a phage display cDNA library. J. Mol. Biol. 297:89-97.

8. Fossa, A., L. Alsoe, R. Crameri, S. Funderud, G. Gaudernack, and E.B. Smeland. 2004. Serological cloning of cancer/testis antigens expressed in prostate cancer using cDNA phage surface display. Cancer Immunol. Immunother. 53:431-438.

9. Konthur, Z. and R. Crameri. 2003. Highthroughput applications of phage display in proteomics analysis. Targets 2:261-270.

10. Crameri, R. and R. Kodzius. 2001. The powerful combination of phage surface display of cDNA libraries and high throughput screening. Comb. Chem. High Throughput Screen. 3:145-155.

11. Konthur, Z., M. Hust, and S. Dübel. 2005. Perspectives for systematic in vitro antibody generation. Gene 364:19-29.

12. Seehaus, T., F. Breitling, S. Dübel, I. Klewinghaus, and M. Little. 1992. A vector for the removal of deletion mutants from antibody libraries. Gene 114:235-237.

13. Faix, P.H., M.A. Burg, M. Gonzales, E.P. Ravey, A. Baird, and D. Larocca. 2004. Phage display of cDNA libraries: enrichment of cDNA expression using open reading frame selection. BioTechniques 36:1018-1029.

14.Zacchi, P., D. Sblattero, F. Florian, R. Marzari, and A.R. Bradbury. 2003. Selecting open reading frames from DNA. Genome Res. 13:980-990.

15. Rondot, S., J. Koch, F. Breitling, and S. Dübel. 2001. A helper phage to improve single-chain antibody presentation in phage display. Nat. Biotechnol. 19:75-78.

16. Sambrook, J., E.F. Fritsch, and T. Maniatis. 1989. Molecular Cloning: A Laboratory Manual, 2nd ed. CSH Laboratory Press, Cold Spring Harbor, NY.

17. Kirsch, M., M. Zaman, D. Meier, S. Dübel, and M. Hust. 2005. Parameters affecting the display of antibodies on phage. J. Immunol. Methods 301:173-185.

18. Voss, S. and A. Skerra. 1997. Mutagenesis of a flexible loop in streptavidin leads to higher affinity for the Strep-tag II peptide and improved performance in recombinant protein purification. Protein Eng. 10:975-982.

19. Kolaskar, A.S. and P.S. Tongaonkar. 1999. A semi-empirical method for prediction of antigenic determinants on protein antigens. FEBS Lett. 276:172-174.

20. Martelli, P.L., P. Fariselli, A. Krogh, and R. Cassadio. 2002. A sequence-profile-based HMM for predicting and discriminating beta barrel membrane proteins. Bioinformatics 18: S46-S53

21. Vieira, J. and J. Messing. 1987. Production of single-stranded plasmid DNA. Methods Enzymol. 153:3-11.

22. Koch, J., F. Breitling, and S. Dübel. 2000 Rapid titration of multiple samples of filamentous bacteriophage (M13) on nitrocellulose filters. BioTechniques 29:1196-1198

23. Carcamo, J., M.W. Ravera, R. Brissette, 0. Dedova, J.R. Beasley, A. Alam-Moghe, C. Wan, A. Blume, and W. Mandecki. 1998 Unexpected frameshifts from gene to expressed protein in a phage-displayed peptide library. Proc. Natl. Acad. Sci. USA 95:1114611151.

24. Goldmann, E., M. Korus, and W. Mandecki. 2000. Efficiencies of translation in three reading frames of unusual nonORF sequences isolated from phage display. FASEB J. 14:603-611.

25. Hust, M. and S. Dübel. 2004. Mating antibody phage display with proteomics. Trends Biotechnol. 22:8-14. 


\section{Research Reports}

26. Crameri, R. and M. Suter. 1993. Display of biologically active proteins on the surface of filamentous phages: a cDNA cloning system for the selection of functional gene products linked to the genetic information responsible for their production. Gene 137:69-75.

27. Paschke, M. and W. Höhne. 2004. A twinarginine translocation (Tat)-mediated phage display system. Gene 350:79-88.

28. Paschke, M. 2006. Phage display systems and their applications. Appl. Microbiol. Biotechnol. 70:2-11.

Received 3 April 2006; accepted 6 June 2006.

Address correspondence to Michael Hust, Technische Universität Braunschweig, Institut für Biochemie und Biotechnologie, Abteilung Biotechnologie, Spielmannstr. 7, 38106 Braunschweig, Germany. e-mail: m.hust@tu-bs.de

To purchase reprints of this article, contact: Reprints@BioTechniques.com 